\title{
Diagnostic and Therapeutic Challenges of Rare Urogenital Cancers: Urothelial Carcinoma of the Renal Pelvis, Ureters and Urethra
}

\author{
Ayun Cassell III ${ }^{\mathrm{a}, \mathrm{b}}$, Burgess Manobah ${ }^{\mathrm{a}}$, Soeghen Willie ${ }^{\mathrm{a}}$
}

\begin{abstract}
Urothelial carcinoma (UC) is a neoplastic growth that affects the lining of the urinary tract from the renal pelvis to the distal urethra. Urothelial cancer occurs less commonly along the upper urinary tract (renal pelvis and ureter) accounting for 5-10\% and even rarer along the urethra approximately less than $1 \%$. The incidence of UC of the upper urinary tract and urethra has been reported in the United States and Europe by the Surveillance, Epidemiology, and End Results Program and the Rare Cancers in Europe project, respectively. Considering the rarity of upper tract urothelial carcinoma (UTUC) and primary urethral cancer (PUC), there is a paucity of data from SubSaharan Africa. Both the European Association of Urology guideline and the National Comprehensive Cancer Network guideline have provided some clinical updates on the management of UTUC and PUC. However, UTUC and PUC present mostly at a more advanced stage than UC of bladder. A high index of suspicion is necessary for diagnosis even more for UTUC. Organ-sparing surgery is possible for both localized UTUC and PUC but stringent follow-up with urine cytology, endoscopy and imaging is mandated for early detection of recurrence.
\end{abstract}

Keywords: Endoscopy; Guidelines; Primary urethral cancer; Rare; Urogenital malignancies; Urothelial cancer of the upper tract

\section{Introduction}

The epithelial lining of the renal calyx, renal pelvis, ureters, bladder and urethra are all embryologically made of urothelium [1]. Urothelial carcinoma (UC) is a neoplastic growth that affects the lining of the urinary tract from the renal pelvis to the distal urethra. UC is the fourth most common malignancy

Manuscript submitted February 1, 2021, accepted February 11, 2021

Published online February 24, 2021

aDepartment of Surgery, John F. Kennedy Medical Center, Monrovia, Liberia ${ }^{\mathrm{b}}$ Corresponding Author: Ayun Cassell, Department of Surgery, John F. Kennedy Medical Center, Monrovia, Liberia. Email: ayuncasselliii@gmail.com

doi: https://doi.org/10.14740/wjon1360
[2] with $90-95 \%$ being bladder cancer [3]. However, UC occurs less commonly along the upper urinary tract (renal pelvis and ureter) accounting for 5-10\% [4] and even rarer along the urethra approximately less than $1 \%[5]$.

In Europe, a pathology is designated rare if there is a prevalence of less than 50/100,000 population [5]. Comparative age-standardized incidence of UC of the renal pelvis, ureters and urethra has been lacking. To date, none of these rare urogenital malignancies has been notified by GLOBOCAN. Rare urogenital cancers are usually inadequately diagnosed due to the low index of suspicion and lack of clinical expertise. The project Surveillance of Rare Cancers in Europe (RARECARE) has now considered epidemiological studies for the documentation of rare cancers in Europe.

Nonetheless, in 2012, a review by Visser et al estimated the age-standardized ratio (ASR) of UC of the renal pelvis and ureters at 14.8/million in males and 6.4 in females. UC of the urethra had a much lower ASR of 1.2/million in men and 0.2/ million in women [4]. The study revealed that the incidence of $\mathrm{UC}$ of the renal pelvis, ureter and urethra was two to three times higher in males compared to females.

Both the Surveillance, Epidemiology, and End Results (SEER) Program and the RARE-CARE project in Europe documented that $\mathrm{UC}$ of the urethra is the most predominant histological type of urethral cancer (54-65\%) followed by squamous cell carcinoma (SCC) (16-22\%) and adenocarcinoma (AC) (10-16\%) [6]. Likewise, UC accounts for nearly $90 \%$ of the histology upper urinary tract tumors with SCC accounting for $<10 \%[2]$.

A population-based study in the USA by Schwarz et al reported an ASR of PUC in the USA at 4.3/million in males and $1.5 /$ million in females, with rates two times higher in blacks than in whites [7]. The exact incidence in Africa is unknown as cancer registries lack data on these rare urogenital malignancies. We cannot postulate that similar incidence in AfricanAmerican males should be extrapolated to African males due to disparity in the risk factors. Even population- and hospital-based studies have underreported UC of the renal pelvis, ureters and urethra in Africa. The cost of diagnosis of UC per patient is the highest of all malignancies due to the required imaging and procedures [8]. This could also preclude the diagnosis of upper tract and urethral cancer in most parts of Africa considering the rarity as well.

The review comparatively analyzes the diagnostic and 
therapeutic challenges of UC of the renal pelvis, ureters and urethra and projects a lapse in diagnosis in Africa, even though rare.

\section{Methodology}

A thorough review of publications was conducted from 1990 to 2020 using the academic search engine and databases including PubMed, Google Scholar, African Journal Online and Google. Both French and English literatures were explored using the Medical Search Heading (MeSH): "rare urogenital malignancies", "upper tract urothelial cancer" and "primary urethral cancer" appended with the following indexes: United States, Europe, Asia, Africa and Sub-Saharan Africa.

The retrieved search items were reviewed for epidemiology, clinical presentation, risk factors, diagnostic imaging, staging (Table 1) [2, 6, 9-15], neoadjuvant treatment, surgery, adjuvant treatment, systemic therapy, prognosis and follow-up (Table 2) $[2,6,9,10,15]$. The references of these publications were also assessed for related articles.

Upper tract urothelial carcinoma (UTUC) was classified according to the tumor-node-metastasis (TNM) 2017 classification [2]. The tumors were classified as papillary urothelial neoplasia of low malignant potential, low grade or high grade, based on the World Health Organization 2004/2016 histological classification. Primary urethra cancer (PUC) in men and women was classified according to the seventh edition of the TNM classification and urothelial PUC was graded into papillary urothelial neoplasm of low malignant potential (PUNLMP), low grade and high grade, according to the $2004 \mathrm{WHO}$ grading system [6].

A qualitative analysis of the data was performed emphasizing comparison in diagnostic and therapeutic challenges between UTUC (renal pelvis and ureter) and PUC of the urethra. The evidence synthesized was reflected in Tables 1 and 2 and in the "Discussion" of the review.

\section{Evidence Synthesis/Results}

After adequate cross-check and vetting for duplication, a total of 34 articles were retrieved which included systematic reviews and meta-analysis, review articles, prospective studies, retrospective studies and international guidelines.

Search results on UTUC included the European Association of Urology (EAU) guideline on UTUC [2] and the European Society of Medical Oncology (ESMO) guidelines for UTUC [9].

Additionally, search results on PUC included the EAU guidelines [6] on PUC and the National Comprehensive Cancer Network (NCCN) guideline on UC [10]. There was no published American Urological Association guideline on PUC or UTUC.

Results from the searched literature could not produce any epidemiological study or guideline on UC of the upper urinary tract and urethra in Africa.

\section{Discussion}

\section{Epidemiology and risk factors of $\mathrm{UC}$ of the renal pelvis and ureter}

The incidence of UTUC peaks in ages of 70 - 90 years and three times more common in males than in females $[2,4]$. Tumors occurring in along the renal calyces and renal pelvis are twice as common as tumors found in the ureters. Carcinoma in situ (CIS) in the upper urinary tract may exist in $11-36 \%$ of individuals and UTUC can be multifocal in $10-20 \%$ of patients [16]. Over $60 \%$ of UTUCs are muscle invasive at diagnosis and about $17 \%$ of cases are synchronous with a bladder tumor [16].

Both hereditary and environmental risk factors have been linked to the development of UTUC. Evidence has shown that familial UTUC has a strong association with hereditary nonpolyposis colorectal cancer [17]. Other established risk factors include the use of tobacco which increases the relative risk. Aristocholic acid found in Chinese herbs is associated with a genetic mutation which causes a nephropathy (Balkan endemic nephropathy) that is a known risk factor for UTUC [11]. Before the 1960s, exposure to certain carcinogenic aromatic amines like benzidine and b-naphthalene was known to cause UTUC until their use was banned [2].

Current data on the epidemiology of UTUC in Sub-Saharan Africa are lacking. A 10-year retrospective review of 548 genitourinary malignancies at the Korle Bu Hospital in Ghana reported only one UTUC and one SCC of the urethra accounting for less than $0.4 \%$ of all malignancies [12]. Moreover, a 15-year study by Bowa et al in Zambia evaluating 8,829 patients with genitourinary malignancies reported other rare urogenital malignancies (testicular cancer and penile cancer) but with no documentation of UTUC [13]. The paucity of data on UTUC in Sub-Saharan Africa could be due to the diagnostic challenge posed by the disease. A high index of suspicion is required to diagnose UTUC confounded by the relatively high cost of endoscopic equipment and computed tomography (CT) scan.

\section{Diagnostic evaluation of $\mathrm{UC}$ of the renal pelvis and ureter}

The diagnosis of UTUC is usually incidental or following a high index of suspicion from presenting symptoms. The commonest presentation is hematuria in $70-80 \%$ of patients $[2,18]$. Flank pain is may be present in $20 \%$ of cases, and $10 \%$ of locally advanced diseases may have lumbar mass. Patients with constitutional symptoms of night sweat, anorexia, weight loss, fever and cough should be assessed for metastatic disease [2].

The diagnostic gold standard for UTUC is CT urography with a sensitivity of $67-100 \%$ and a specificity of $93-99 \%$ [2, 14]. The disadvantage of CT scan is that it may not detect flat epithelial lesion. Findings of hydronephrosis are worrisome and denote advanced disease (Fig. 1a, b).

Patients who are contraindicated to CT urography due to iodinated contrast media can undergo magnetic resonance im- 


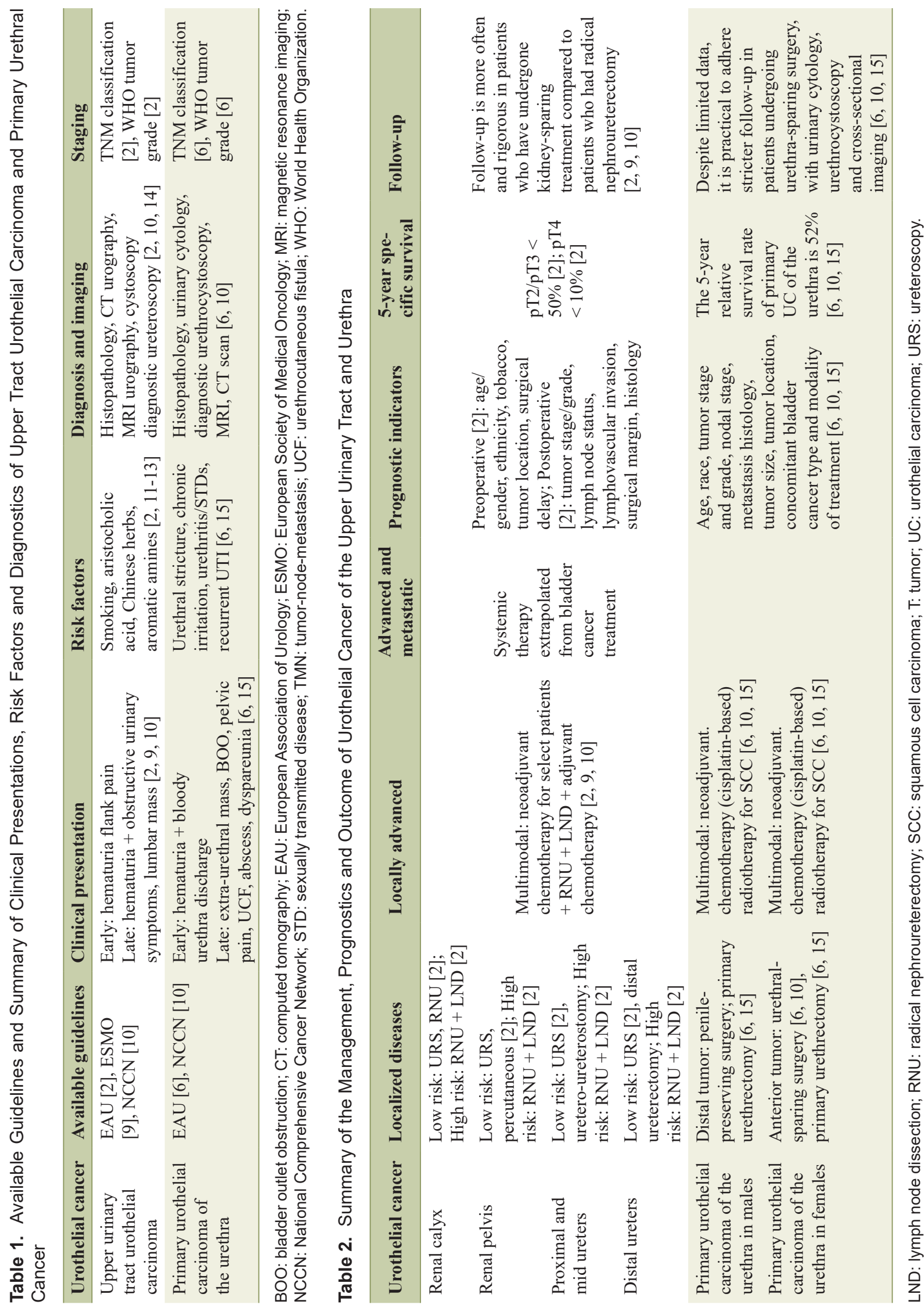



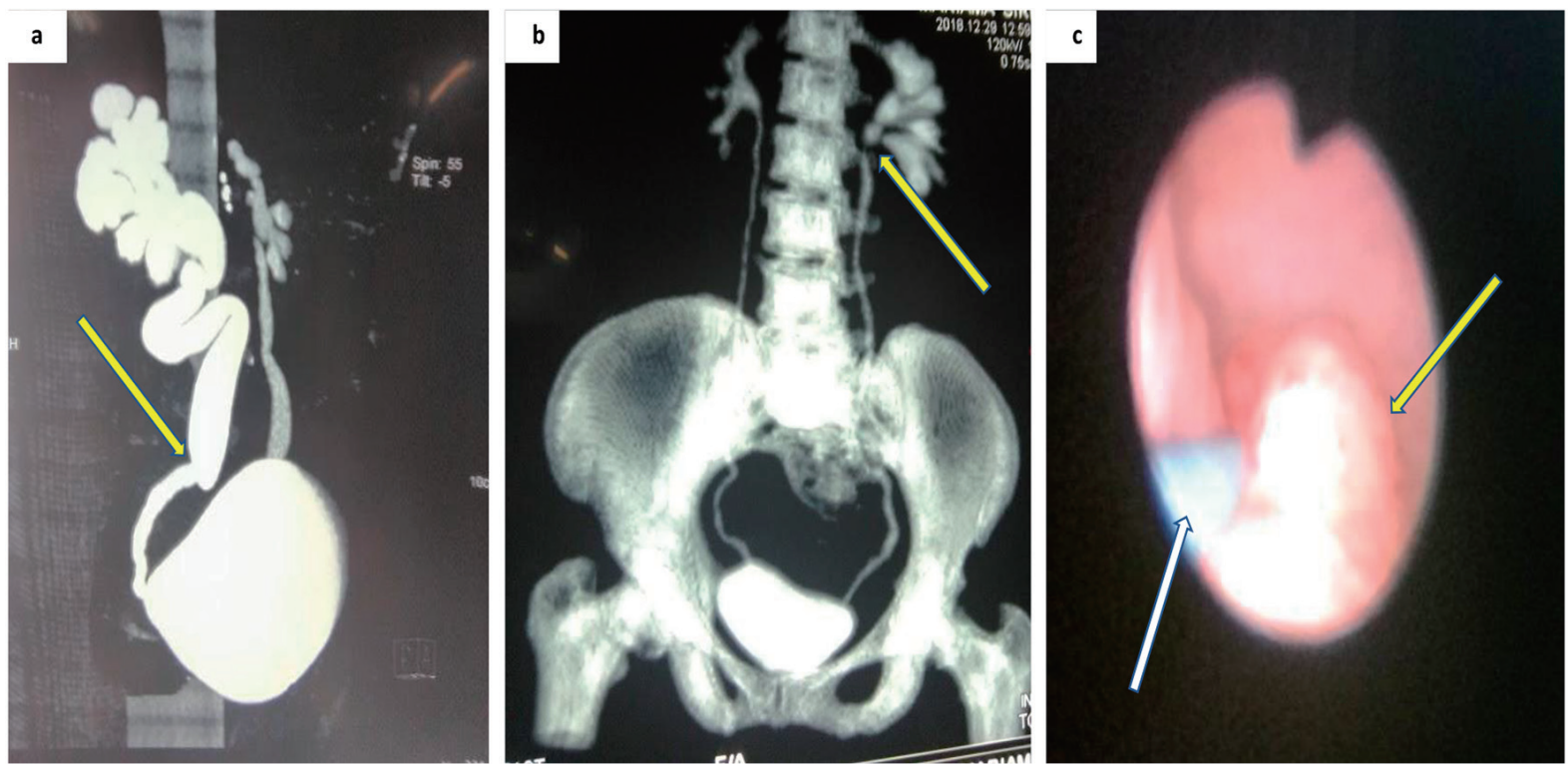

Figure 1. Imaging of upper urinary tract tumor. (a) CT urography showing suspicion zone of stenosis causing right unilateral hydroureteronephrosis. (b) Coronal view CT urography with filling defect in the proximal left ureters. (c) Ureteroscopy showing a non-obstructing tumor along mid ureters (white arrow showing the guidewire). CT: computed tomography.

aging (MRI) urography [14]. However, patients with severe renal failure are at risk of nephrogenic systemic fibrosis due to gadolinium-based contrast media [2].

Cystoscopy is performed to rule out bladder cancer. A normal cystoscopy and positive urine cytology are suggestive of UTUC if there is no evidence of CIS of the bladder or urethra.

Suspicious lesions on imaging can be biopsied using flexible ureteroscopy (URS) [2]. Patients with solitary kidney or those opting for nephron-sparing surgery are best investigated with flexible URS (Fig. 1c).

\section{Risk stratification and prognosis of UTUC}

Locally advanced UTUC has a very poor prognosis ranging from $50 \%$ to less than $10 \%$ [19]. The EAU 2017 guideline update on UTC outlined several prognostic factors including preoperative and postoperative factors. Preoperative prognostic indicators include age, gender, ethnicity, tobacco use, tumor location and surgical delay, while the postoperative factors were tumor grade and stage, lymph node status, lymphovascular invasion, surgical margins, histopathology, molecular markers and bladder recurrence.

UTUCs are difficult to stage clinically before surgery. However, the use of risk stratification as low risk or high has guided the decision of nephron-sparing surgery or radical nephroureterectomy (RNU). Based on the WHO grading system, patients with low risk disease are known to have unifocal disease, tumor size $<2 \mathrm{~cm}$, low-grade cytology, low-grade URS biopsy and no invasive aspect on CT urography [2]. Characteristics of high-risk UTUC in patients included hydronephrosis, tumor size $>2 \mathrm{~cm}$, high-grade cytology, high-grade URS biopsy, multifocal disease, previous radical cystectomy for bladder cancer and variant histology [2].

\section{Management of UTUC}

Management of low risk/localized UTUC

The EAU and NCCN UTUC guidelines recommend patients with low risk UTUC can benefit from kidney-sparing surgery (KSS) if the oncological outcome will not be compromised [2, 10]. KSS is also advocated in patients with solitary kidney or diseased contralateral kidney. Low risk tumor along the ureters may be managed by endoscopic laser ablation using flexible URS $[2,9,10]$. However, tumor found along the renal pelvis and lower calyxes may be approached by percutaneous access but with documented risk of tumor seeding [2]. Low risk tumors found in the distal ureters can undergo complete distal ureterectomy and ureteroneocystostomy. Segmental resection for tumors along the mid and proximal ureter is possible but has higher failure rates than resection of the distal ureters $[2$, $9,10]$.

\section{Adjuvant instillation}

There is some evidence to suggest that adjuvant instillation of bacillus Calmette-Guerin (BCG) vaccine or mitomycin C along the upper urinary tract has oncological benefit for KSS and CIS $[2,9,10]$. Instillation can be performed through a percutaneous nephrostomy tube or retrograde instillation through a ureteric stent.

\section{Management of high risk/locally advanced UTUC}

The gold standard for treatment of high-risk disease is RNU 
and excision of the bladder cuff because of a considerable risk of tumor recurrence in this area. There is strong evidence to suggest that adjuvant instillation of mitomycin within $72 \mathrm{~h}$ of surgery can reduce the recurrence rate within the first year [2, $9,10]$.

According to the ESMO guideline, neoadjuvant chemotherapy may be reasonable in this setting for few reasons. The patient has both kidneys present which offer better tolerability of the drugs. Secondly, the additional oncological benefit of downstaging the tumor can offer better prognostication [9]. Nevertheless, due to the lack of appropriate preoperative pathological specimen to stage muscle invasion, patients may be subjected to toxic drugs for a rather low risk disease.

Some data are now reporting that in safe hands, laparoscopic RNU has the potential to provide similar oncological outcome to open RNU [20]. High risk patients with T2-T4 disease should undergo lymph node dissection (LND) because of the increased possibility of nodal involvement. A systemic review of anatomical landmark for LND recommended dissection of the renal hilar, precaval, pericaval and retrocaval nodes for right-sided tumors of the renal pelvis, upper and middle third of the ureter, while for left-sided tumors, the renal hilar, preaortic and paraaortic nodes should be dissected [21].

\section{Advanced/metastatic UTUC}

The management of advanced and metastatic UTUC is multimodal as RNU has shown no oncological benefit except for palliation. There are insufficient data on systemic therapy for metastatic UTUC. The EAU and the NCCN guidelines on UTUC recommend the use of cisplatin-based regimen extrapolated from the treatment of metastatic urothelial bladder cancer. Unfortunately, not all patients are candidate for systemic therapy. Cisplatin-based chemo-regimen is associated with considerable nephrotoxicity [3]. Moreover, most of these patients with metastatic UTUC have concurrent deteriorating renal function and might not benefit from systemic therapy [2, 3].

\section{1) Management of UTUC in Sub-Saharan Africa}

There is insufficient evidence to depict the current standard of care of UTUC in Africa. A case series by Niang et al in 2014 reported four cases of upper tract tumor managed by RNU and excision of the bladder cuff [22]. However, the use of postoperative instillation was not documented. One of the patients with T3 disease had recurrence at the ureteral stump with nodal and visceral metastasis after 2 months. Another patient with $\mathrm{T} 1$ disease had recurrence at the perimeatal bladder cuff and underwent endoscopic resection.

\section{2) Follow-up for UTUC}

There is risk of $22-47 \%$ of recurrence of bladder cancer in pa- tients treated for UTUC and 2-6\% in the contralateral upper tract. Therefore, the EAU guideline recommends rigorous surveillance following organ-sparing procedures for UTUC with cystoscopy, URS and upper tract imaging at 3- to 12-month intervals. Patients who underwent RNU for UTUC can be screened using cystoscopy, URS, urine cytology and CT scan at varying intervals depending on the risk assessment $[2,9,10]$.

\section{Epidemiology and risk factors of urothelial PUC}

The peak incidence of PUC is almost similar to UTUC. According to an analysis by the SEER Program, the peak incidence of PUC was in the $>75$ years age group (7.6/million) and almost negligible in ages $<55$ years [6]. Like UTUC, approximately $50 \%$ of symptomatic patients with PUC will have locally advanced disease and about $40-60 \%$ of patients with muscle invasive bladder cancer may later develop urethral cancer [15].

These advanced presentations can be alluded to the diagnostic challenge posed by the location of these tumors especially UTUC or the relative thin wall layers of the ureters and urethra risking faster muscle invasion. In Africa, the lack of diagnostic tool, poor health seeking behavior and poverty have been a barrier to both diagnosis and treatment of UCs [23]. Contemporary reviews from Sub-Saharan Africa have revealed much later stages of presentation of other rare genitourinary cancers including penile and testicular tumor [24, 25]. To date, the available literature on UC of the upper tract or urethra remains sparse. A 10-year retrospective study evaluating the histological pattern of urological malignancies in Calabar, South-Southern Nigeria found a total of 617 urological malignancies but only two cases of PUC were reported (one UC and one AC) accounting for $0.3 \%$ of urological malignancies [26].

Numerous etiological factors have been reported for PUC in males, including urethral stricture, chronic irritation from catheterization and chronic urethral inflammation from sexually transmitted diseases $[6,15]$. In women, recurrent urinary tract infection and urethral diverticula have been associated with PUC.

\section{Diagnostic evaluation of urothelial PUC}

Early symptoms of PUC include visible hematuria or bloody urethral discharge. In locally advanced disease, extra-urethral mass, urethra-cutaneous fistula, bladder outlet obstruction, pelvic pain, abscess and dyspareunia are common findings [15]. A detailed genital exam should be performed both in males and females. The clinical characteristic of inguinal nodes bilaterally should be assessed and documented.

Urethrocystoscopy and biopsy of the urethral lesion are fundamental for diagnosis $[6,10]$. In patients with UC of the prostatic urethra, transurethral resection at 5 and 7 o'clock distally to the verumontanum produces better diagnostic yield [6, $10,15]$. MRI is essential to assess the extent of the tumor locally or the response to neoadjuvant chemotherapy [27]. CT scan of the abdomen and thorax is done to assess distant spread. 
The presence of enlarged lymph node following imaging may portend metastatic disease.

\section{Prognosis of PUC}

The mean 1- and 5-year survivals of PUC in Europe according to the RARECARE Project are $71 \%$ and $54 \%$, respectively [4]. According to EAU 2013 guidelines on PUC, the mean predictors of survival of PUC were race, age, tumor grade and stage, nodal status, distant metastasis, histological characteristics, tumor location, tumor size, concomitant bladder cancer and type of treatment modality. Additionally, an SEER data analysis by Aleksic et al from 2004 to 2010 revealed that the only significant predictors of cancer-specific survival (CSS) were age and the stage of PUC [28]. An SEER database analysis from 1988 to 2006, involving 2,065 men with PUC by Rabbani et al, revealed that age, grade, tumor stage, histological type and extent of surgery were predictive of overall survival and CSS [29]. UC was associated with better outcome than SS but worse outcome than AC.

\section{Management of PUC (UC)}

\section{Treatment of PUC in males}

According to the EAU guidelines on PUC, penile-preserving surgeries are currently replacing penile amputation for male distal urethral cancer [6]. Findings from retrospective data have shown similar oncological outcome with distal urethrectomy for anterior urethral tumor compared to penile amputation [30]. Additional inguinal or iliac LND can be performed for individuals with clinically suspicious lymph nodes. However, the NCCN guideline on PUC recommends repeat transurethral resection for Tis, Ta, or T1 disease of the proximal or prostatic urethra followed by intraurethral instillation with BCG, mitomycin, or gemcitabine [10]. Males with T2 tumors along the bulbar urethra may undergo urethrectomy and cystoprostatectomy [10]. There is limited evidence to support the use of neoadjuvant or adjuvant at this stage. If the patient is staged T3, T4 or has nodal involvement after surgery, adjuvant chemotherapy should be considered. Both the NCCN and EAU guidelines recommend neoadjuvant chemotherapy for T3 and T4 UC of the urethra followed by urethrectomy + cystoprostatectomy $[6,10]$. Cisplatinbased chemotherapeutic regimen is the recommended choice. Another alternative to surgery is chemoradiation but with benefits shown only in SCC of the urethra [6]. Both male and female patients with advanced or metastatic PUC of the urethra should be treated with similar regimen as metastatic bladder cancer using gemcitabine plus cisplatin or high dose intensity methotrexate, vinblastine, doxorubicin, and cisplatin (MVAC) plus granulocyte-colony-stimulating factor (GCSF) $[3,6]$.

\section{Treatment of PUC in females}

Initial treatments of females with $\mathrm{T} 2$ include urethrectomy with cystectomy as urethral-sparing surgery is associated with high local recurrence rate [10]. Primary radical urethrectomy includes the removal of all the periurethral tissue from the bulbocavernosus muscle bilaterally, with a cuff of all adjacent soft tissue up to the pubic symphysis and bladder neck. Chemoradiation is another option but has considerable side effects like urethral stenosis, fistula, necrosis, and cystitis and/or hemorrhage [6]. The NCCN guideline recommends that females with T3 and T4 UC of the urethra can benefit from neoadjuvant chemotherapy (MVAC) followed by sure by primary radical urethrectomy $[6,10]$. Patients with recurrent disease can undergo pelvic exenteration which includes en bloc removal of the bladder, urethra and anterior vagina + urinary diversion [10].

\section{Follow-up for PUC}

There are insufficient data to properly outline a follow-up algorithm for PUC. As such, the EAU guideline recommends a stringent surveillance after organ-sparing procedures for PUC. A 10-year retrospective study by Gakis et al reported that recurrence in patients treated for PUC was significantly associated with advanced nodal stage, advanced pathological tumor and proximal tumor location [31].

It seems reasonable to advocate a more extensive followup with urinary cytology, urethrocystoscopy and cross-sectional imaging in patients treated for PUC.

\section{Management of PUC in Sub-Saharan African}

To date, data remain scarce on the epidemiology and management of PUC in Africa. The only available data are few case series or case reports. In 2018, Kouka et al in Senegal reported two cases of PUC in females, an infiltrating $\mathrm{AC}$ and a UC, respectively [32]. Both cases were locally advanced on presentation and underwent pelvic exenteration and ileal conduit. In 2012, Gowe et al in Ivory Coast reported a case of metastatic PUC (T3N3M1) who was offered emasculation and systemic chemotherapy. The patient refused care and died within 6 months [33]. Though the disease may be rare, it is also apparent that the few cases that may erupt are either left undiagnosed due to diagnostic challenges, poor health seeking behaviors of patients or lack of access to specialized care. There is remarkable shortage of specialists in the Sub-Saharan region and most facilities' diagnostic capabilities are underequipped.

\section{Future direction}

More genomic study is required for UC to direct systemic therapy in the future. Randomized control trials are difficult to conduct for UTUC and PUC due to the rarity of the disease. In Africa where resource is limited, focus should be placed on training of cancer specialist, creation of cancer centers equipped with provision of endoscopic equipment, CT scans and MRI for diagnosis, staging and management of genitourinary cancer. However, a multidisciplinary approach with the 
limited human and material resource available is necessary to augment the diagnostic capabilities for cancer care. The few urologists, medical oncologists, surgical oncologists, radiologists and radiotherapists have to collaborate to formulate guideline adopted for resource limited settings [34].

\section{Conclusion}

UC represents a broad spectrum of diseases occurring rarely along the upper urinary tract and urethra. Despite the rarity of UTUC and PUC, there is also paucity of data from Sub-Saharan Africa. Both EAU and NCCN guidelines have provided some clinical updates on the management of UTUC and PUC. However, UTUC and PUC present mostly at a more advanced stage than UC of bladder. A high index of suspicion is necessary for diagnosis even more for UTUC. Organ-sparing surgery is possible for both localized UTUC and PUC but stringent follow-up with urine cytology, endoscopy and imaging is mandated for early detection of recurrence.

\section{Acknowledgments}

Special thanks were given to the Department of Surgery, Liberia College of Physicians and Surgeons.

\section{Financial Disclosure}

None to declare.

\section{Conflict of Interest}

The authors declare no conflict of interest concerning this paper.

\section{Author Contributions}

All authors have made considerable inputs to conception and design, acquisition of data, analysis and interpretation of data; engaged in organizing the article or revising it analytically for relevant intellectual content; gave final consent of the version to be published; and agree to be responsible for all aspects of the work.

\section{Data Availability}

The authors declare that data supporting the findings of this study are available within the article.

\section{References}

1. Miyazaki J, Nishiyama H. Epidemiology of urothelial carcinoma. Int J Urol. 2017;24(10):730-734.

2. Roupret M, Babjuk M, Comperat E, Zigeuner R, Sylvester RJ, Burger M, Cowan NC, et al. European Association of urology guidelines on upper urinary tract urothelial carcinoma: 2017 update. Eur Urol. 2018;73(1):111-122.

3. Babjuk M, Bohle A, Burger M, Capoun O, Cohen D, Comperat EM, Hernandez V, et al. EAU Guidelines on non-muscle-invasive urothelial carcinoma of the bladder: update 2016. Eur Urol. 2017;71(3):447-461.

4. Visser O, Adolfsson J, Rossi S, Verne J, Gatta G, Maffezzini M, Franks KN, et al. Incidence and survival of rare urogenital cancers in Europe. Eur J Cancer. 2012;48(4):456464.

5. Gatta G, van der Zwan JM, Casali PG, Siesling S, Dei Tos AP, Kunkler I, Otter R, et al. Rare cancers are not so rare: the rare cancer burden in Europe. Eur J Cancer. 2011;47(17):2493-2511.

6. Gakis G, Witjes JA, Comperat E, Cowan NC, De Santis M, Lebret T, Ribal MJ, et al. EAU guidelines on primary urethral carcinoma. Eur Urol. 2013;64(5):823-830.

7. Swartz MA, Porter MP, Lin DW, Weiss NS. Incidence of primary urethral carcinoma in the United States. Urology. 2006;68(6):1164-1168.

8. Botteman MF, Pashos CL, Redaelli A, Laskin B, Hauser $\mathrm{R}$. The health economics of bladder cancer: a comprehensive review of the published literature. Pharmacoeconomics. 2003;21(18):1315-1330.

9. Leow JJ, Chong KT, Chang SL, Bellmunt J. Upper tract urothelial carcinoma: a different disease entity in terms of management. ESMO Open. 2016;1(6):e000126.

10. Flaig TW, Spiess PE, Agarwal N, Bangs R, Boorjian SA, Buyyounouski MK, Chang S, et al. Bladder Cancer, Version 3.2020, NCCN clinical practice guidelines in oncology. J Natl Compr Canc Netw. 2020;18(3):329-354.

11. Colin P, Koenig P, Ouzzane A, Berthon N, Villers A, Biserte J, Roupret M. Environmental factors involved in carcinogenesis of urothelial cell carcinomas of the upper urinary tract. BJU Int. 2009;104(10):1436-1440.

12. Klufio GO. A review of genitourinary cancers at the Korle-Bu teaching hospital Accra, Ghana. West Afr J Med. 2004;23(2):131-134.

13. Bowa K, Kachimba JS, Labib MA, Mudenda V, Chikwenya M. The changing pattern of urological cancers in Zambia. Medical Journal of Zambia. 2008;35(4):157-159.

14. Cowan NC, Turney BW, Taylor NJ, McCarthy CL, Crew JP. Multidetector computed tomography urography for diagnosing upper urinary tract urothelial tumour. BJU Int. 2007;99(6):1363-1370.

15. Janisch F, Abufaraj M, Fajkovic H, Kimura S, Iwata T, Nyirady P, Rink M, et al. Current disease management of primary urethral carcinoma. Eur Urol Focus. 2019;5(5):722-734.

16. Cosentino M, Palou J, Gaya JM, Breda A, RodriguezFaba O, Villavicencio-Mavrich H. Upper urinary tract urothelial cell carcinoma: location as a predictive factor for concomitant bladder carcinoma. World J Urol. 2013;31(1):141-145.

17. Roupret M, Yates DR, Comperat E, Cussenot O. Upper urinary tract urothelial cell carcinomas and other urologi- 
cal malignancies involved in the hereditary nonpolyposis colorectal cancer (lynch syndrome) tumor spectrum. Eur Urol. 2008;54(6):1226-1236.

18. Froemming A, Potretzke T, Takahashi N, Kim B. Upper tract urothelial cancer. Eur J Radiol. 2018;98:50-60.

19. Lughezzani G, Burger M, Margulis V, Matin SF, Novara G, Roupret M, Shariat SF, et al. Prognostic factors in upper urinary tract urothelial carcinomas: a comprehensive review of the current literature. Eur Urol. 2012;62(1):100114.

20. Latchamsetty KC, Porter CR. Treatment of upper tract urothelial carcinoma: a review of surgical and adjuvant therapy. Rev Urol. 2006;8(2):61-70.

21. Campi R, Minervini A, Mari A, Hatzichristodoulou G, Sessa F, Lapini A, Sessa M, et al. Anatomical templates of lymph node dissection for upper tract urothelial carcinoma: a systematic review of the literature. Expert Rev Anticancer Ther. 2017;17(3):235-246.

22. Niang L, Kouka C, Diallo Y, Ndoye M, Kane R, Jalloh M, Ndiaye A, Labou I, Diaw J, Gueye SM. Primary tumors of the upper urinary tract: report of 4 cases. Open Journal of Urology. 2014;4(09):115-120.

23. Cassell A, Yunusa B, Jalloh M, Mbodji MM, Diallo A, Ndoye M, Diallo Y, et al. Non-muscle invasive bladder cancer: a review of the current trend in Africa. World J Oncol. 2019;10(3):123-131.

24. Cassell A, Jalloh M, Ndoye M, Yunusa B, Mbodji M, Diallo A, Gaye O, et al. Review of testicular tumor: diagnostic approach and management outcome in Africa. Res Rep Urol. 2020;12:35-42.

25. Cassell A, Yunusa B, Manobah B, Wambo D. Management guidelines of penile cancer- a contemporary review of sub-Saharan Africa. Infect Agent Cancer. 2020;15:26.

26. Isiwele EM, Bassey IA, Ikpi EE, Enakirerhi GE, Otobo
FO, Essiet A, Ekwere PD. Histopathologic patterns of urological malignancies in Calabar, South-Southern Nigeria: a ten-year review. Journal of Cancer and Tumor International. 2018;8(1):1-10.

27. Dayyani F, Hoffman K, Eifel P, Guo C, Vikram R, Pagliaro LC, Pettaway C. Management of advanced primary urethral carcinomas. BJU Int. 2014;114(1):25-31.

28. Aleksic I, Rais-Bahrami S, Daugherty M, Agarwal PK, Vourganti S, Bratslavsky G. Primary urethral carcinoma: A Surveillance, Epidemiology, and End Results data analysis identifying predictors of cancer-specific survival. Urol Ann. 2018;10(2):170-174.

29. Rabbani F. Prognostic factors in male urethral cancer. Cancer. 2011;117(11):2426-2434.

30. Smith Y, Hadway P, Ahmed S, Perry MJ, Corbishley CM, Watkin NA. Penile-preserving surgery for male distal urethral carcinoma. BJU Int. 2007;100(1):82-87.

31. Gakis G, Morgan TM, Efstathiou JA, Keegan KA, Mischinger J, Todenhoefer T, Schubert T, et al. Prognostic factors and outcomes in primary urethral cancer: results from the international collaboration on primary urethral carcinoma. World J Urol. 2016;34(1):97-103.

32. Kouka SC, Diallo Y, Jalloh M, Bentefouet L, Ly R. Two cases of female urethral primary cancer: review of clinico pathological and therapeutic aspects. Med Sur Urol. 2018;7(211):1-3.

33. Gowe EE, Dekou AH, Vodi CC, Konan PG, Gnagne MY, Dje K. Carcinome urothelial de l'uretre: une nouvelle observation et revue de la litterature. Basic and Clinical Andrology. 2012;22(4):268-271.

34. Cassell A, Jalloh M, Yunusa B, Ndoye M, Mbodji MM, Diallo A, Kouka SC, et al. Management of renal cell carcinoma-current practice in Sub-Saharan Africa. J Kidney Cancer VHL. 2019;6(2):1-9. 